\title{
LOCALLY COMPACT TOPOLOGIES FOR GROUPS $\left(^{1}\right)$
}

\author{
BY \\ NEIL W. RICKERT
}

The investigations leading to this paper were suggested by the papers of Hewitt [3] and Ross [5]. In [3] Hewitt was interested in proving that if an abelian group is locally compact in two topologies, one strictly stronger than the other, there is a character continuous in one topology and discontinuous in the other (actually a special case of a theorem of Kaplansky-see Theorem 1.1 of [2]). Actually Hewitt proved a stronger result. His arguments were based on the fact that both the additive group of reals, and the multiplicative group of complex numbers of absolute value 1 have the property that every stronger locally compact group topology is discrete. A natural question to ask is what other groups have this property. The answer is very simple ( 2.1 of this paper). We consider in the second section of this paper the obvious generalization. Namely which groups have the property that there are only finitely many stronger locally compact group topologies. The investigations in the first section of this paper were suggested by the paper of Ross [5]. Ross was considering the same question as Hewitt, and was led to consider the relationship between two locally compact group topologies on a group $G$ such that $G$ has the same closed subgroups in the two topologies. We investigate this further in the first section of this paper, and are able to say that many of the properties of $G$ as a topological group can be recovered once we know the closed subgroups.

1. Closed subgroups of locally compact groups. We consider here what we can say about a locally compact group $G$ once we know its closed subgroups. Throughout this section of the paper $R$ will denote the additive group of real numbers, $T$ will denote the multiplicative group of complex numbers of absolute value 1 , and, except for $R$ all our groups will be written multiplicatively.

1.1. TheOREM. The closed subgroups of $G$ determine the open subgroups of $G$.

Proof. In case $G$ is abelian, the proof is very easy, for then a subgroup is open if and only if every subgroup containing it is closed. In the nonabelian case this does not characterize the open subgroups (every subgroup of $S L(3, C)$ containing $S U(3)$ is closed, and in fact is either $S U(3)$ or $S L(3, C)$, but $S U(3)$ is not open). Thus for the general case we proceed by the following steps.

(1) The closed subgroups of $G$ determine the identity component $G_{0}$ of $G$. In fact $G_{0}$ is the intersection of the closed subgroups $H$ with the property that for

Received by the editors December 1, 1965.

(1) The research leading to this paper was partially supported under grant NSF-GP-3509. 
every closed subgroup $K$ and every subgroup $L$ for which $H \cap K \subset L \subset K, L$ is closed.

(2) A closed subgroup $H$ of $G$, with $H \supset G_{0}$ has the property that $H / G_{0}$ is compact if and only if for each closed subgroup $K$ with $G_{0} \subset K \subset H$ and every closed subgroup $L$ of $K$ such that $L$ is of countable index in $K, L$ is of finite index in $K$.

(3) A subgroup $L$ of $G$ is open if and only if it is closed, and $L \cap H$ is of finite index in $H$ whenever $H$ is a closed subgroup containing $G_{0}$ such that $H / G_{0}$ is compact.

1.2. COROLlaRY. The closed subgroups determine the identity component of $G$. If $G$ is totally disconnected, the closed subgroups determine the topology of $G$.

Proof. The identity component of $G$ is the intersection of the open subgroups. If $G$ is totally disconnected the open subgroups form a neighborhood basis at the identity.

We remark that in the special case where $G$ is compact abelian and totally disconnected, this result was proved by Ross in [5].

1.3. COROLlaRY. The closed subgroups determine whether $G$ is o-compact or whether $G$ is compactly generated.

Proof. $G$ is $\sigma$-compact if and only if every open subgroup is of countable index. $G$ is compactly generated if and only if there is a finite subset $F$ of $G$, and an open subgroup $H$ of $G$ with $H / G_{0}$ compact, such that $G$ is generated by $F \cup H$.

1.4. THEOREM. The closed subgroups determine whether $G$ is finite dimensional. If $G$ is solvable and finite dimensional the closed subgroups determine the dimension of $G$.

Proof. $G$ is finite dimensional if and only if every strictly decreasing chain of closed connected subgroups of $G$ is of finite length. In case $G$ is solvable, the dimension of $G$ is one less than the maximum length of such a chain.

\subsection{THEOREM. The closed subgroups of $G$ determine whether $G$ is a Lie group.}

Proof. $G$ is a Lie group if and only if $G$ is finite dimensional and every closed totally disconnected subgroup of $G$ is discrete (discreteness is characterized by the trivial subgroup being open).

\subsection{THEOREM. The closed subgroups of $G$ determine whether $G$ is compact.}

Proof. $G$ is compact if and only if every open subgroup of $G$ is of finite index, and $G$ does not contain a closed infinite cyclic subgroup.

We remark that this theorem was proved by Ross in [5] in the abelian case.

1.7. LemMA. Let $G$ be a group which we know to be isomorphic to $R$ as a topological group. Then the continuous automorphisms of $G$ determine the topology of $G$.

Proof. Fix an $x$ in $G$. Call an element $y$ of $G$ positive if and only if there is a continuous automorphism $\lambda$ of $G$ such that $y=\lambda(\lambda(x)$ ). (Thus if we think of $x$ as being $1, y$ is positive if and only if it is a square.) The set of positive elements determines an ordering of $G$, and the topology of $G$ is the order topology. 
1.8. THEOREM. The closed subgroups of $G$ determine whether $G$ is a connected twodimensional Lie group.

Proof. $G$ is a connected two-dimensional Lie group if and only if $G$ is a connected solvable two dimensional Lie group.

1.9. THEOREM. If $G$ is a noncompact connected two-dimensional Lie group, the closed subgroups of $G$ determine the topology of $G$.

Proof. Case I. $G$ is not abelian. In this case $G$ is a semidirect product of a normal subgroup $N$ and a subgroup $L$, both of which are one-dimensional noncompact Lie groups (i.e., isomorphic to $R$ ). But then the continuous automorphisms of $N$ are those which are inner automorphisms in $G$ generated by elements of $L$. Thus Lemma 1.7 applies, and the topology of $N$ is determined. But then the topology of $L$ is determined as the topology of the automorphism group of $N$. Finally the topology of $G$ is the product topology.

Case II. $G$ is abelian. In this case we can find closed subgroups $H$ and $K$ of $G$ which are closed in $G$, such that $H$ is a one-dimensional noncompact connected Lie group, and $K$ is a one-dimensional Lie group, and $G=H \times K$. But then an automorphism $\lambda$ of $H$ is continuous if and only if for every homomorphism $\alpha$ of $H$ into $K$, the subgroup consisting of elements $(x, \alpha(x))$ is closed if and only if the subgroup consisting of elements $(x, \alpha(\lambda(x)))$ is closed. Thus once again we may use Lemma 1.7 to see that the topology of $H$ is determined. But then a homomorphism $\alpha$ of $H$ into $K$ is continuous if and only if the subgroup consisting of elements $(x, \alpha(x))$ is closed. Thus we see that the closed subgroups of $G$ determine also the topology of $K$, and thus the topology of $G$ as the product topology.

1.10. COROLLARY. If $G$ is a noncompact connected solvable Lie group, the topology of $G$ is determined by the closed subgroups of $G$, providing $G$ is at least two-dimensional.

Proof. If $G$ is abelian this follows from 1.9 by an obvious argument. For the nonabelian case an easy induction argument works.

1.11. THEOREM. If $G$ is a product of closed subgroups $H$ and $K$ each of which is a one-dimensional compact connected Lie group, the topology is determined by the topology of $H$ together with the closed subgroups of $G$.

Proof. An isomorphism $\alpha$ of $H$ onto $K$ is continuous if and only if the subgroup consisting of elements $(x, \alpha(x))$ is closed. Thus the topology of $K$ is determined, and so is the topology of $G$.

1.12. TheOREM. If $G$ is a nonabelian connected Lie group the topology of $G$ is determined by the closed subgroups of $G$.

Proof. This follows easily from 1.10, 1.11, and a theorem of Freudenthal [1]. 
1.13. COROLlary. If the identity component of $G$ is not abelian the topology of $G$ is determined by the closed subgroups.

Proof. If $G$ is a Lie group this follows immediately from 1.12. The general case follows from the fact that $G$ has an open subgroup which is an inverse limit of its Lie factor groups.

Henceforth we shall concern ourselves only with abelian groups.

1.14. THEOREM. If $G$ is abelian, the identity component of $G$ is not compact, and $G$ is not one dimensional, the topology of $G$ is determined by the closed subgroups of $G$.

Proof. For every compact subgroup $K$ of $G$ such that $G / K$ is a Lie group of dimension at least two, the topology of $G / K$ is determined by the closed subgroups of $G / K$ (and hence by the closed subgroups of $G$ ) as follows easily from 1.10 . The result now follows since $G$ is the inverse limit of such factor groups.

1.15. TheOREM. Let $G$ be a locally compact abelian group whose identity component is compact, and let $\chi_{0}$ be a continuous character on $G$ which is not constant on the identity component of $G$. Let $\chi$ be any other character on $G$. Then $\chi$ is continuous if and only if it satisfies one of the following conditions.

(a) There is a character $\chi_{1}$ with closed kernel, and there are integers $n$ and $m$ such that both $\chi_{1}^{n} \chi_{0}$ and $\chi_{1}^{m} \chi$ have their kernels open.

(b) The kernel $K$ of $\chi$ is closed, $G /(K \cap H)$ is a two-dimensional compact connected Lie group, where $H$ is the kernel of $\chi_{0}$, and the kernel of $\chi \chi_{0}$ is closed.

In particular, $\chi_{0}$ and the closed subgroups of $G$ determine the continuous characters on $G$.

Proof. The last assertion follows from the first. The proof of the first assertion is a routine one, provided that one realizes that an isomorphism from $T$ to $T$ is continuous if its graph is a closed subgroup of $T \times T$. We omit the details.

1.16. THEOREM. If the identity component of $G$ is compact, if a character which is continuous, and is not constant on the identity component is known, and if the closed subgroups of $G$ are known, the topology of $G$ is known.

Proof. From 1.15 we see that we know all the continuous characters on $G$. There is a compact open subgroup $K$ of $G$. But then the topology of $K$ is the smallest topology in which the continuous characters of $G$ are continuous on $K$. But since $K$ is open, this also determines the topology of $G$.

1.17. THEOREM. If the identity component of $G$ is compact, the closed subgroups of $G$ determine the character group of $G$ as an abstract group, and they determine the closed subgroups of the character group of $G$. In fact we may construct a subgroup $L$ of the group $M$ of all (not necessarily continuous) characters on $G$, such that there is an (in general discontinuous) automorphism $\lambda$ of $T$ with the property that the 
automorphism of $M$ defined by $\chi \rightarrow \lambda \circ \chi$ takes $L$ onto the (continuous) character group of $G$.

Proof. From our assumptions there is a closed subgroup $H$ of $G$ such that $G / H$ is a compact connected one-dimensional Lie group. Let $\chi_{0}$ be any character on $G$ whose kernel is $H$ ( $\chi_{0}$ need not be continuous). Let $L$ be the set of characters $\chi$ which satisfy either condition (a) or condition (b) of the statement of Theorem 1.15. From our assumptions on $H$ we know that there is an automorphism $\lambda$ of $T$, such that $\lambda \circ \chi_{0}$ is a continuous character on $G$. But then it is obvious from 1.15 that the automorphism of $M$ defined by $\chi \rightarrow \lambda \circ \chi$ takes $L$ onto the (continuous) character group of $M$. In particular $L$ is a group, and $L$ is isomorphic to the character group of $G$ as an abstract group. It is easily seen that a subgroup of $L$ corresponds to a closed subgroup of the (continuous) character group of $G$ under the automorphism of $M$ if and only if this subgroup is the intersection of kernels of elements of $G$ (considered as characters on $L$ ).

This theorem tells us what the possible continuous character groups are, provided we know the closed subgroups. Our next result shows that in the compact case, any such group can be realized as the group of continuous characters under some topology with the same closed subgroups.

1.18. TheOREM. Let $G$ be a compact abelian group under a topology $\tau$. Let $\lambda$ be a (discontinuous) automorphism of $T$. Then there is a topology $\tau_{1}$ on $G$ under which $G$ is a compact group with the same closed subgroups, and such that a character $\chi$ is $\tau$-continuous if and only if $\lambda \circ \chi$ is $\tau_{1}$-continuous.

Proof. This is obvious if $G$ is the product of copies of $T$ (with $\tau$ the product topology). The general case follows from the fact that $G$ can be embedded as a closed subgroup of a product of copies of $T$.

We now consider a question asked by Ross in [5]. He asked whether the closed subgroups of $G$ determine $G$ as a topological group up to isomorphism.

1.19. TheOREM. The closed subgroups of $G$ determine $G$ up to isomorphism as a locally compact group if $G$ satisfies any one of the following conditions:

(i) The identity component of $G$ is not compact.

(ii) $G$ is compactly generated.

(iii) For every $x$ in $G$ the smallest closed subgroup containing $x$ is compact (in particular if $G$ is compact).

Proof. (i) If $G$ is not one dimensional, this follows from 1.14. If $G$ is one dimensional, its identity component $G_{0}$ is a one-dimensional noncompact connected ?... group, so its topology is determined up to isomorphism. But in this case $G$ is a product of its identity component and a totally disconnected group, so an appeal to 1.2 completes the proof.

(ii) This follows from cases (i) and (iii) together with the structure theorem.

(iii) In this case the identity component is compact, so we may apply 1.17 and 
see that up to isomorphism the character group of $G$ is known, and furthermore the closed subgroups of the character group are determined. But our assumptions imply that the character group of $G$ is totally disconnected, so an application of 1.2 shows that the character group of $G$ as a locally compact group is determined up to isomorphism. The result now follows from the duality theorem.

1.20. REMARK. It is easy to conclude from what we have proved that the set of continuous characters on $G$ determines the topology of $G$ as a locally compact group (since the closed subgroups are then determined as being those which are intersections of kernels of continuous characters). We omit the details, since in any case a stronger result is already known (Theorem 1.1 of [2]).

For the remainder of this section of the paper we will assume that $G$ is a locally compact abelian group under each of two topologies $\tau_{1}$ and $\tau_{2}$. We assume furthermore that every $\tau_{1}$-closed subgroup is $\tau_{2}$-closed.

1.21: THEOREM. Every $\tau_{1}$-open subgroup is $\tau_{2}$-open.

Proof. If $H$ is $\tau_{1}$-open, every subgroup containing $H$ is $\tau_{1}$-closed, so every subgroup containing $H$ is $\tau_{2}$-closed, so $H$ is $\tau_{2}$-open.

1.22. THEOREM. If $G$ is $\tau_{2}$-compact it is $\tau_{1}$-compact.

Proof. If $H$ is $\tau_{1}$-open it is $\tau_{2}$-open, so of finite index. Furthermore $G$ does not contain a $\tau_{1}$-closed infinite cyclic subgroup, since otherwise it would contain a $\tau_{2}$-closed infinite cyclic subgroup.

1.23. THEOREM. If $G$ is $\tau_{2}$-compact, the $\tau_{1}$-identity component and the $\tau_{2}$-identity component are identical.

Proof. Let $H$ be the $\tau_{1}$-identity component. Obviously the $\tau_{2}$-identity component is contained in $H$ (this follows from 1.21). On the other hand, since $H$ is $\tau_{1}$-connected it is divisible. Thus in the topology $\tau_{2}, H$ is compact and divisible, from which it follows that $\mathrm{H}$ is $\tau_{2}$-connected, thus completing the proof.

1.24. Theorem. If $G$ is $\tau_{1}$-totally disconnected, $\tau_{2}$ is stronger than $\tau_{1}$. If $G$ is $\tau_{2}$ compact and $\tau_{2}$-totally disconnected, $\tau_{1}$ and $\tau_{2}$ are identical.

Proof. This follows immediately from 1.21, 1.22, and 1.23.

If $\tau_{2}$ is actually stronger than $\tau_{1}$, and if $G$ is $\tau_{2}$-compact, it follows that $\tau_{1}$ and $\tau_{2}$ are identical, so every $\tau_{2}$-closed subgroup is $\tau_{1}$-closed. One might ask whether always if $G$ is $\tau_{2}$-compact every $\tau_{2}$-closed subgroup is $\tau_{1}$-closed. The example below shows this fails.

1.25. ExAmple. Denote by $K$ the compact abelian group whose character group is the additive rational numbers with the discrete topology. Since $K$ is divisible and torsion free, $K$ is an abstract group, a direct sum of copies of the rationals. Likewise $T$ as an abstract group is a direct sum of its torsion subgroup and copies of the rationals. A simple cardinality argument then shows that as abstract groups $T$ and $T \times K$ are isomorphic. Let $\rho$ be an isomorphism of $T$ onto 
$T \times K$. Define the topology $\tau_{1}$ on $T$ to be the usual topology, and define $\tau_{2}$ to be the topology which is induced from the usual topology on $T \times K$ via the isomorphism $\rho$. Since the only $\tau_{1}$-closed subgroups are the finite subgroups, we see that every $\tau_{1}$-closed subgroup is $\tau_{2}$-closed. However, even though the group is $\tau_{2^{-}}$ compact, there is a subgroup (for example $\rho^{-1}(K)$ ) which is $\tau_{2}$-closed but not $\tau_{1}$-closed.

2. Stronger topologies for locally compact abelian groups. In this section of the paper, which is independent of the earlier part of the paper, we consider the question of which locally compact abelian groups have the property that the only stronger topology under which the group is a locally compact group is the discrete topology. This turns out to have a simple solution. We then consider, and solve, the problem of determining which locally compact abelian groups have the property that there are only finitely many stronger topologies in which it is a locally compact group.

Notation. Since we shall only be concerned with abelian groups we shall always use additive notation, and shall denote the zero element of the group by 0 . For a locally compact group $G$ we will denote the connected component of the identity by $G_{0}$, and we will denote the group of continuous characters by $G^{\wedge}$. Unless we specifically mention otherwise all our groups will be locally compact abelian. $R$ is the usual group of real numbers, $T$ is the unit circle of the complex plane under multiplication (but we shall write the operation as addition), and $Z_{p}$ ( $p$ a prime) is the additive group of integers of the $p$-adic field. We recall that $Z_{p}$ is a locally compact integral domain.

2.1. THEOREM. Let $G$ be a locally compact abelian group. Then every stronger locally compact group topology on $G$ is discrete if and only if $G$ is discrete, or $G$ contains an open subgroup isomorphic to either $T, R$, or $Z_{p}$.

Proof. If $G$ contains an open subgroup isomorphic to either $T$ or $R$ every strictly stronger topology in which $G$ is a locally compact group is well known to be discrete, since both $T$ and $R$ are Lie groups. Suppose $G$ contains as an open subgroup the group $Z_{p}$. We might as well assume that $G$ is $Z_{p}$. Clearly in any stronger topology $G$ is totally disconnected, so there is a compact open subgroup $K$. If $K$ is the identity, it follows that the stronger topology is the discrete topology. If it is not the identity, we will show it is open in $Z_{p}$, and hence the stronger topology on $G$ is the same as the original topology. We must show that every closed subgroup of $Z_{p}$ is open (or equivalently, is of finite index). By passing to the character group this is obvious. For the converse, suppose that every stronger locally compact topology on $G$ is discrete. Suppose that $H$ is a closed subgroup of $G$. If $H$ is neither open nor discrete, we could give $G$ a strictly stronger nondiscrete topology by giving $H$ its relative topology, and topologizing $G$ so as to make $H$ an open subgroup. It follows then that every closed subgroup of $G$ is either open or discrete. Then if $G$ is not totally disconnected, its identity component is open. In this case 
there is therefore no loss of generality in assuming $G$ connected. But then there is a nontrivial character on $G$, and its kernel must be discrete, so it is a local isomorphism of $G$ onto $T$. Thus $G$ is either $T$ or $R$. (More correctly $G$ contains either $T$ or $R$ as an open subgroup.) In the totally disconnected case, by passing to an open subgroup if necessary, we may assume $G$ is compact. If every element of $G$ is of finite order, an easy application of the Baire category theorem shows that $G$ is of bounded order. Thus $G^{\wedge}$ is of bounded order, and hence a sum of finite groups. Correspondingly $G$ is a product of finite groups and if it is not a finite sum there is a closed subgroup which is neither open nor discrete. This is impossible, so in this case $G$ is discrete. Otherwise $G$ contains an element of infinite order and hence a compact monothetic infinite subgroup, which must necessarily be open. Thus we may assume that $G$ is monothetic. By writing $G$ as a product of groups corresponding to the primary decomposition of $G^{\wedge}$ we see that $G$ contains $Z_{p}$ as an open subgroup for some prime $p$ (see p. 408 of [4]). This completes the proof.

2.2. LemMA. Let $p_{1}, p_{2}, \ldots, p_{n}$ be distinct primes. Let $H$ be a closed subgroup of $Z_{p_{1}} \times Z_{p_{2}} \times \cdots \times Z_{p_{n}}$.Then

$$
H=\left(H \cap Z_{p_{1}}\right) \times\left(H \cap Z_{p_{2}}\right) \times \cdots \times\left(H \cap Z_{p_{n}}\right) .
$$

Furthermore, for each $i, H \cap Z_{p_{i}}$ is either the trivial group, or is open and isomorphic to $Z_{p_{i}}$.

Proof. Denote by $H^{\perp}$ the subgroup of the character group of $Z_{p_{1}} \times Z_{p_{2}} \times \cdots$ $\times Z_{p_{n}}$ consisting of those characters which vanish identically on $H$. Then the proof is easily completed by taking the primary decomposition of $H^{\perp}$.

2.3. LEMMA. If $G$ is a compact abelian group which is a product of infinitely many nontrivial compact groups, we may find for each real number $r$ a compact subgroup $G_{r}$ such that for $r<r^{\prime}, G_{r}<G_{r^{\prime}}$ and $G_{r^{\prime}} / G_{r}$ is infinite. Consequently we may find for each $r$ a locally compact group topology on $G$, stronger than the original topology of $G$, such that for $r<r^{\prime}$, the topology corresponding to $r$ is strictly stronger than the topology corresponding to $r^{\prime}$.

Proof. The second assertion follows from the first, for we may define the topology corresponding to $r$ by declaring $G_{r}$ to be open, while giving $G_{r}$ the relative topology as a subgroup of $G$. For the first assertion, observe that we may assume, by combining factors if necessary, that $G$ is a product (that is direct product) of countably many subgroups. We may index these subgroups by the rationals. Thus suppose they are denoted by $H_{q}$ (for $q$ a rational). Then we define $G_{r}$ to be the subgroup of $G$ consisting of those elements whose component in $H_{q}$ is zero for every $q>r$. It is easily checked that the lemma is now true.

2.4. Corollary. Let $G$ be an infinite compact abelian group in which every element is of finite order. Then the number of strictly stronger topologies has at least the power of the continuum. 
Proof. Such a group is an infinite direct product of finite groups (see p. 406 of [4]).

2.5. CoROllary. Let $G$ be a compact abelian group, and suppose that in the primary decomposition of $G^{\wedge}$ there are infinitely many nontrivial factors. Then the number of strictly stronger locally compact group topologies on $G$ has at least the power of the continuum.

Proof. Under these conditions $G$ is an infinite direct product.

2.6. LemMA. Let $G$ be a locally compact group of dimension at least two. Then the number of strictly stronger locally compact group topologies has at least the power of the continuum.

Proof. $G$ has a factor group which is a Lie group of dimension at least two. Since one-parameter subgroups of this factor group may be lifted to one-parameter subgroups of $G$, it is easily seen that the number of distinct one-parameter subgroups of $G$ has at least the power of the continuum. For each such one-parameter subgroup, we may define a locally compact group topology on $G$ by making the one-parameter subgroup a one-dimensional Lie group, and topologizing $G$ so as to make this subgroup open.

2.7. Lemma. Let $p$ be a prime. Then the number of distinct locally compact group topologies on $Z_{p} \times Z_{p}$ stronger than the usual topology has at least the power of the continuum.

Proof. For each element $\alpha$ in $Z_{p}$, let $H_{\alpha}$ be the subgroup of $Z_{p} \times Z_{p}$ consisting of elements $(z, \alpha z)$ (recall that $Z_{p}$ is an integral domain). Define a topology $\tau_{\alpha}$ by giving $H_{\alpha}$ its relative topology, and topologize $Z_{p} \times Z_{p}$ so as to make $H_{\alpha}$ open. It is easily checked that the topologies $\tau_{\alpha}$ are all distinct and clearly their cardinality is that of the continuum.

2.8. LemMA. Let $G$ be an infinite compact abelian group, such that every element of $G^{\wedge}$ has order a power of $p$ for some fixed prime $p$. Suppose that the cardinality of the set of locally compact group topologies stronger than the given topology is less than the power of the continuum. Then $G$ contains an open subgroup isomorphic to $Z_{p}$.

Proof. From 2.4 we see that $G$ has an element $z$ of infinite order. Let $H$ be the closed subgroup it generates. But $H$ is isomorphic to $Z_{p}$ (see p. 408 of [4]). We claim that $H$ is open. If not, the group $G / H$ is infinite and compact. As in the proof of 2.4 we see that $G / H$ has an element of infinite order. Let $y$ be an element of $G$ which maps onto an element of infinite order in $G / H$. Then it is easily seen that the closed subgroup of $G$ generated by $y$ and $z$ is isomorphic to $Z_{p} \times Z_{p}$, so 2.7 gives us the desired contradiction, so $H$ is indeed open, and the proof is complete.

We now have enough information at our disposal to determine which are the locally compact abelian groups with only finitely many stronger locally compact group topologies. 
2.9. Definition. Let $p_{1}, p_{2}, \ldots, p_{k}$ be distinct primes. We define $K_{p_{1} p_{2} \cdots p_{k}}$ to be the compact abelian group whose dual group is algebraically the additive group of rationals $m / n$ for which $p_{1}, p_{2}, \ldots, p_{k}$ are the only primes dividing $n$.

2.10. TheOREM. A locally compact abelian group $G$ has the property that the set of stronger locally compact group topologies has cardinality less than that of the continuum if and only if $G$ contains as an open subgroup one of the following groups:

(a) The trivial group,

(b) $R$,

(c) $T$,

(d) $Z_{p_{1}} \times Z_{p_{2}} \times \cdots \times Z_{p_{n}}\left(p_{1}, p_{2}, \ldots, p_{n}\right.$ distinct primes $)$,

(e) $K_{p_{1} p_{2} \cdots p_{n}}\left(p_{1}, p_{2}, \ldots, p_{n}\right.$ distinct primes),

(f) $R \times Z_{p_{1}} \times \cdots \times Z_{p_{n}}\left(p_{1}, p_{2}, \ldots, p_{n}\right.$ distinct primes $)$,

(g) $T \times Z_{p_{1}} \times \cdots \times Z_{p_{n}}\left(p_{1}, p_{2}, \ldots, p_{n}\right.$ distinct primes $)$,

(h) $K_{p_{1} \cdots p_{s}} \times Z_{p_{s+1}} \times \cdots \times Z_{p_{n}}\left(p_{1}, \ldots, p_{n}\right.$ distinct primes $)$.

In these cases the number of stronger topologies is
(a) 1
(b) 2
(c) 2
(d) $2^{n}$
(e) $2^{n+1}$
(f) $2^{n+1}$
(g) $2^{n+1}$
(h) $2^{n+1}$.

Proof. We first establish the formula for the number of stronger topologies in cases (a), .., (h). Using the structure theorem (p. 90 of [4]), and similar arguments to those in the proof of 2.1 it is easily seen that in cases (a), (b), (c), (d), (f) every stronger topology is obtained by taking a closed subgroup (which can be chosen to be a product of some of the direct factors of the group) giving the subgroup its relative topology, and topologizing the group so as to make the subgroup open. The formula for the number of stronger topologies then follows. In cases (e), (g), (h) the groups are factor groups of the group in (f) by an infinite cyclic subgroup, and are thus locally isomorphic. It is easily seen that the stronger topologies are in one-one correspondence with the stronger topologies of the group in case ( $f$ ), and the formula follows. Suppose now that the number of stronger topologies on $G$ has cardinality less than the power of the continuum. Consider first the case where $G$ is totally disconnected. By passing to an open subgroup if necessary, we may assume that $G$ is compact. We may apply 2.5 to conclude that in the primary decomposition of $G^{\wedge}$ there are only finitely many nontrivial factors. By applying 2.8 to each of the corresponding factors of $G$ we see that $G$ has an open subgroup $Z_{p_{1}} \times Z_{p_{2}} \times \cdots \times Z_{p_{n}}$. We next consider the case when $G$ is connected. We are assured on account of 2.6 that $G$ is one dimensional. If $G$ is either $R$ or $T$ we are 
done. Otherwise we may find a closed totally disconnected subgroup $K$ such that $G / K$ is isomorphic to $T$. Every stronger topology on $K$ yields a stronger topology on $G$ since we can always topologize $G$ so that $K$ is open. Thus from the case we have considered, $K$ is isomorphic to $Z_{p_{1}} \times Z_{p_{2}} \times \cdots \times Z_{p_{n}}$ (more precisely, an open subgroup has this form, but we may always replace $K$ by such an open subgroup). By considering the character group of $G$ it is easily deduced that $G$ is isomorphic to $K_{p_{1} p_{2} \cdots p_{n}}$. Next we consider the case that the identity component of $G$ is not compact. By passing to an open subgroup if necessary we may assume that $G$ is isomorphic to $R \times K$ where $K$ is a totally disconnected group (because of $2.6 G$ is one-dimensional). Applying the totally disconnected case to the group $K$ we see that $G$ contains as an open subgroup the group of case (f). Consider now the case where the identity component of $G$ is isomorphic to $T$. Since $T$ is injective (its character group is projective) we see that it is a direct summand, and the arguments we have used for the previous case can be adapted to show that $G$ has as an open subgroup the group of case (g). Finally we consider the case that the identity component is compact, nontrivial, and not isomorphic to $T$. If the identity component is open this reduces to the connected case. We assume then that $G_{0}$ is not open. By passing to an open subgroup if necessary we may assume that $G$ is compact. Again there is a compact subgroup $K$ of $G$ such that $G / K$ has an open subgroup isomorphic to $T$. Again, from the totally disconnected case, we know that $K$ is isomorphic to $Z_{p_{1}} \times Z_{p_{2}} \times \cdots \times Z_{p_{n}}$. By passing to an open subgroup of $K$ and renumbering the $p_{t}$ if necessary, we see that $G_{0} \cap K$ is isomorphic to $Z_{p_{1}} \times Z_{p_{2}} \times \cdots \times Z_{p_{s}}$ for some integer $s<n$. Thus we conclude that $G_{0}$ is isomorphic to $K_{p_{1} p_{2} \cdots p_{s}}$ exactly as in the connected case, and furthermore we conclude that $G$ has an open subgroup isomorphic to $G_{0} \times Z_{p_{s+1}} \times \cdots \times Z_{p_{n}}$. This completes the proof.

2.12. RemarK. I have not thought much about this, but it does seem likely that the same techniques can be used to prove that if an abelian group is a locally compact group under two topologies $\tau_{1}$ and $\tau_{2}$ with $\tau_{2}$ stronger than $\tau_{1}$, the number of locally compact group topologies lying between the two topologies $\tau_{1}$ and $\tau_{2}$ is either finite, or has at least the power of the continuum.

\section{REFERENCES}

1. H. Freudenthal, Die Topologie der Lieschen Gruppen als algebraisches Phänomen. I, Ann. of Math. 42 (1941), 1051-1074.

2. I. Glicksberg, Uniform boundedness for groups, Canad. J. Math. 14 (1962), 269-276.

3. E. Hewitt, $A$ remark on characters of locally compact abelian groups, Fund. Math. 53 (1963), 55-64.

4. E. Hewitt and K. A. Ross, Abstract harmonic analysis. I, Springer, Berlin, 1963.

5. K. A. Ross, Closed subgroups of locally compact abelian groups, Fund. Math. 56 (1965), 241-244.

YALE UNIVERSITY,

New Haven, Connecticut 